\title{
Papers
}

\section{Treatments of homosexuality in Britain since the 1950s-an oral history: the experience of professionals}

\author{
Michael King, Glenn Smith, Annie Bartlett
}

\begin{abstract}
Objective To investigate the experiences of professionals who administered and evaluated treatments for homosexuality in Britain since the 1950s.

Design A nationwide study based on qualitative interviews. Participants 30 health professionals who developed and practised treatments for homosexuality.

Results A range of treatments were developed to make homosexuals into heterosexuals, the most common of which were behavioural interventions. Treatments were based on little evidence of effectiveness and were open to the criticism that legal or social pressures coerced patients. Treatments did not become mainstream within British mental health services. With hindsight, professionals realised that they had not appreciated the influence of social context on sexual behaviour. Most now regarded same sex attraction as compatible with psychological health, although a small minority considered that the option to try to become heterosexual should still be available to patients who desire it.

Conclusions Social and political assumptions sometimes lie at the heart of what we regard as mental pathology and serve as a warning for future practice.
\end{abstract}

\section{Introduction}

Legal sanctions against homosexual behaviour together with prejudice against gay men and lesbians rose to a peak after the second world war and laid the foundation on which interest in psychological interventions to alter sexuality increased sharply in the $1960 \mathrm{~s}$ and $70 \mathrm{~s}^{\mathrm{s}^{-3}}$ In our companion paper (Online First on bmj.com) we describe the experiences of patients who underwent treatments to change their sexual orientation in Britain from the 1950s onwards. Here we examine the motivations and experiences of professionals who developed and practised these treatments and place them in the context of their professional and personal lives and the historical period in which they worked.

\section{Methods}

All participants gave written, informed consent to take part. We had previously identified professionals who had published research in this specialty ${ }^{1}$ and asked each to name other professionals who had administered treatments or were knowledgeable about them. We also used professional contacts, advertisements, articles, and programmes in the national media and names given by participants in the accompanying paper (Online First, bmj.com). GS and MK used tape recorded, in depth interviews to explore professionals' personal and professional backgrounds; how they had become expert with particular treatments; and their attitudes to their work at the time and changes in their attitudes since that time.

\section{Analysis}

As in our earlier paper, ${ }^{2}$ we approached the narratives using a chronological framework that formed the basis for a more detailed analysis. We used the computer software package (NVivo) to break down the material into manageable parts. All authors undertook a series of discussions about emerging themes and atypical cases.

\section{Results}

We identified 44 professionals, of whom 30 (aged 50 to 80 years) agreed to participate. Some refused because they thought the topic was no longer relevant or feared receiving unwanted media attention. Two psychoanalysts had died and one declined to be interviewed. We interviewed 12 psychiatrists, 16 psychologists, one nurse specialist, and one electrician who had developed electric shock equipment. All but two had worked in the NHS.

\section{Life and career before administering treatments}

The Maudsley Hospital in London established behaviour treatments in the 1960s. The emerging discipline of clinical psychology was influenced by seminal work that suggested neurotic disorders were acquired through faulty learning and might respond to behaviour modification. ${ }^{4}$ Clinics for the treatment of homosexuality became established in London, Birmingham, Manchester, Glasgow, and Belfast. Most professionals became involved by accident rather than design.

Well I didn't have much choice. That was a clinical placement. I was [the consultant's] first student. Basically the first year I was there, more or less all I ever did was shove electricity down homosexual patients.

They entered psychology or psych understanding people's behaviour. However, several came from pure science backgrounds and lacked awareness of the social and cultural context of human behaviour. Despite describing open minded family backgrounds, most had grown up in the same era of conservatism about sexuality as their patients. Most encountered gay men and lesbians for the first time as inexperienced young clinicians. They often described how treatments were experimental in nature, with scant regard for efficacy or ethics:

Here were people coming along who seemed to be asking for help, it was against the law, they wanted to change their behaviour, that's how it was presented to us. You never thought about the 
morality of what you were doing. You were effectively a technician. Clinical psychologist

They rarely questioned the prevailing assumption that same sex attraction was abnormal or considered that people could adapt to their sexuality:

I must say that at least at first I was not aware of any particular ethical difficulty. Dealing with it by ameliorating their social background, rather than dealing with their sexual orientation, hadn't really occurred to any of us, certainly not me.

Clinical psychologist

\section{Treatments}

Most of the professionals provided behavioural treatments, which included aversion therapy and covert sensitisation. Aversion therapy with electric shock was the most common treatment:

We had to become electrifying geniuses! The situation was you had the screen, the person sat at the table with the things [equipment] on and with a lever that they had to pull to avoid the shocks. The pictures started off with pretty men, working their way through ugly men into ugly women and into pretty women. That was the whole process literally.

Mental health nurse

Intermittent aversion schedules were commonly used, as it was believed that the new behaviour was less readily extinguished. Professionals' descriptions of treatment corresponded with patients', ${ }^{2}$ although one reported that his patients had several weeks of inpatient assessment, giving the patient time to withdraw from treatment. Talking to patients was believed to compromise the effectiveness of aversion therapy. Other behavioural treatments included covert sensitisation, in which patients would counter homosexual thoughts with shameful fantasies of arrest by the police or discovery by family. Masturbating to a homosexual fantasy and switching to a heterosexual one near orgasm was also advised.

Other treatments described were psychoanalysis and hypnotherapy. Treatments seemed to be used throughout the country with no general protocol or ethical guidelines. Few lesbians received treatment.

One leading advocate of treatments in the 1960s and 1970s reported that he became convinced that helping men to control compulsive homosexual behaviour was the most effective option:

Certainly after 1975 I would tell them [patients] that I didn't think it was possible to change their sexual orientation. The main people I treated were predominantly heterosexual, who felt their homosexual behaviours had become compulsive and they wanted to get them under control.

Psychiatrist

None the less, many spoke of their increasing doubts and dilemmas about the efficacy and ethics of any such treatments:

From the data I looked at, it undoubtedly inhibited their [homosexuals] sexual behaviour and there was loads of evidence of that. They were psychologically castrated if you like-heavy word. But you hadn't put anything in its place.

Clinical psychologist

Many professionals came to recognise the social context of sexual behaviour. One leader in the field was shocked to find his work publicly compared with brain washing and Nazi experimentation. Several eventually considered aversion therapy unjustifiable and pursued "softer" behavioural techniques together with social skills training:

We also had retraining, if that's the word, in sexual and social behaviours because many of these people appeared, at least to us, to be deficient in making sexual advances to women.

\section{Outcomes of treatment}

Outcome assessments were variable, and systematic follow up was attempted only in research settings. There were mixed views about efficacy of treatments but a minority of professionals still regarded treatment as effective:

This young man came in with homosexuality. Completely cured, went out and married a girl who turned out to be lesbian. We treated her, she got better and the last time we heard of them they were married with $4-5$ children!

Psychiatrist

Others considered that gains were more limited but that it was still possible to curb homosexual behaviour:

I think two or three people really had become satisfactorily heterosexual. The rest felt that their problems had been ameliorated in that they were either better disposed to their homosexual condition or the fear that some of them had concerning homosexual behaviour had modified, either because they had been able to reduce it, terminate it, or been able to talk to people and become more adjusted to it.

Clinical psychologist

Most doubted the treatment's efficacy, however, and came to question whether they were acting in patients' best interests. They began to think that treatment was underpinning questionable social values and that patients might say anything to convince them that it had worked to avoid yet more treatment or further legal repercussions:

People were referred from the courts as voluntary patients as an alternative to prison, which isn't terribly voluntary. People were motivated to say things that weren't actually true.

Psychiatrist

\section{Life and career after administering treatments}

None of those interviewed had made treatment of homosexuality their life work. Like their patients, they were influenced by changing public attitudes to sexuality and evolving ideas on the social politics of sexual expression:

With hindsight I look back and say that's just part of the horror stories of the 1950s and $60 \mathrm{~s}$ of general homophobia. The fact that it had a theoretical underpinning was true but essentially an element. Nobody would have thought of using that theory to treat homosexuals had there not been this great big kerfuffle about homosexuality that was still existent. There's no such thing as a totally neutral free profession that does things purely because of their scientific interest.

Clinical psychologist

Several also spoke of their guilt about their use of these treatments, which they now regarded as a form of punishment, and their unease in talking about their involvement with family, friends, and colleagues:

I feel a lot of shame. I don't think I've ever spoken about it since then apart from now. I'm sure I've talked about a lot of the other clinical experiences.

Clinical psychologist

However, a small minority still maintained that same sex attraction is a mental illness requiring treatment or at the least is associated with psychopathology:

I thought they [homosexuals] were people who were disordered and needed treatment and psychiatric help. And I still do.

Clinical psychologist

A few even voiced concern that people who wanted to change were denied the opportunity by the demise of these treatments:

If there was a treatment that could change homosexuality for most people who wanted to change that wouldn't feel unreasonable to me, because I still see guys who are predominantly homosexual but are really very uncomfortable with the whole gay scene. So I could see someone like that, if there was a treatment to make them heterosexual, to give it to them.

Psychiatrist 


\section{Discussion}

Our results show that many professionals were uneasy about the treatments for homosexuality during the time of their involvement. Others realised with hindsight that they lacked understanding of sexual behaviour within its social context. Their work was based on little evidence of effectiveness and was open to the charge that legal or social pressures coerced patients. Some professionals were excited to be in the "vanguard" of mental health, but this enthusiasm blinded them to the dilemmas facing their patients and the damage their treatments might cause. Those who felt troubled by administering these treatments were often compromised by their junior status and lack of autonomy. Although clinics were established in several British cities, treatments did not seem to become mainstream within UK mental health services. Only a small minority believed that current practice denied people distressed by their homosexuality an effective means to change their sexual orientation. Our data show how assumptions about public morality and professional authority can lead to the medicalisation of human differences and the infringement of human rights.

\section{Limitations}

We had a clearer sampling frame for recruiting professionals than we did for patients, ${ }^{2}$ and thus the professionals are probably representative of those who undertook behavioural treatments to change sexual orientation in the NHS in the United Kingdom. However, we cannot claim that they are representative of all those who undertook treatments to change sexual orientation. Although we learned something of psychoanalytical practice from participants in the accompanying paper, we had little opportunity to interview leading psychoanalysts here. As in all oral history studies, we do not know how much the passage of time and changes in social attitudes influenced our participants' recall of events or how much later rationalisation influenced their accounts.

We are aware that other theoretical approaches could have been applied to these qualitative data. For example, our section on "life and career before administering treatments" could also be seen as a theme concerning assumptions about normality and professional authority. Similarly, our section "outcomes of treatment" could be seen as addressing changes in professional and social attitudes. We believe, however, that time lines represent how the history of such treatments unfolded and allow the oral histories to speak for themselves.

\section{Conclusions}

Despite their inherent limitations, narratives such as these may reveal much more than academic opinion or official documents on why society encouraged the use of such treatments on healthy, but unhappy, individuals. Modern medical practice requires an adequate evidence base for treatments and requires that clinicians and members of government consider the adequacy and appropriateness of disease entities that originate from the interplay of scientific and social perspectives. At a time when there is considerable anxiety about government plans to manage people with so called dangerous and severe personality

\section{What is already known on this topic}

Little is known about the personal views and experiences of medical and psychology professionals in the United Kingdom who attempted to make homosexual men and women heterosexual in the 20th century

\section{What this study adds}

Treatments varied throughout the country, with no general protocol or ethical guidelines

Behavioural treatments were most common, including aversion therapy with electrical shock

Though some professionals consider that the treatments were valid, many had increasing doubts about efficacy and ethics

The evolving concepts in the light of liberalisation of public attitudes to homosexuality show how social and moral attitudes can determine what is regarded as "pathology"

disorders, this study reminds us of the risks of ignoring the association between science and society.

We thank all the men and women who participated in the study; BBC Radio Kent, Merseyside, Cornwall, Scotland, Norfolk, Foyle (Northern Ireland), Radio 4's All in the Mind programme, BBC Breakfast Television, Time Out (London), Glasgow Herald, Manchester Evening News, Daily Mail, Gay Times, Diva Magazine, Pink Paper, and many other groups that helped to advertise this study; and Jeffrey Weeks and John Warder, who provided advice at all stages of the study, and Éamonn McKeown for commenting on earlier drafts of this paper. We also acknowledge the support of the Camden and Islington Mental Health and Social Care Trust.

Contributors: MK and $\mathrm{AB}$ conceived the idea for the study, devised the protocol, and obtained funding for the study. MK and GS conducted the interviews and analysed the data. All three authors contributed to the paper. MK is guarantor.

Funding: GS was supported by a grant from the Wellcome Trust History of Medicine Section.

Competing interests: None declared.

Ethical approval: Royal Free Hospital NHS Trust research ethics committee approved the study.

1 King MB, Bartlett A. British psychiatry and homosexuality. $\mathrm{Br} J$ Psychiatry 1999;174:106-13.

2 Smith G, King M, Bartlett A. Treatments of homosexuality in Britain since the $1950 \mathrm{~s}-$ an oral history: the experience of patients. BMJ 2004 doi 10.1136/ bmj.37984.442419.EE

3 Weeks J. Coming out. Homosexual politics in Britain from the nineteenth century to the present. London: Quartet Books, 1990.

4 Wolpe J. Psychotherapy by reciprocal inhibition. Stanford, CA: Stanford University Press, 1958.

(Accepted 2 December 2003)

doi $10.1136 /$ bmj.37984.496725.EE

Department of Mental Health Sciences, Royal Free and University College School of Medicine, Royal Free Campus, London NW3 2PF

Michael King professor of primary care psychiatry

Glenn Smith research fellow

Department of Psychiatry, St George's Hospital Medical School, Jenner Wing, London SW17 0RE

Annie Bartlett senior lecturer

Correspondence to: Michael King m.king@rfc.ucl.ac.uk 